\title{
Pediatric Skull Fractures Based on Three-Dimensional Computed Tomography: Correlation of Skull Fracture Types, Location, Severity, and Accompanying Head Injuries with Surgical Risk in 291 Children
}

\author{
Hyeong Rae Lee ${ }^{1}$, Jong Yeon Kim², Yong Cheol Lim, Soo Han Yoon \\ ${ }^{1}$ Department of Neurosurgery, Ajou University Medical Center, Ajou University School of Medicine, Suwon, \\ ${ }^{2}$ Department of Neurosurgery, Wonju Severance Christian Hospital, Yonsei University Wonju College of Medicine, Wonju, \\ Republic of Korea
}

Corresponding author:

Soo Han Yoon

Department of Neurosurgery, Ajou University Medical Center, Ajou University School of Medicine, 164, World cup-ro, Yeongtong-gu, Suwon 16499, Republic of Korea

Tel: $+82-31-219-5233$

Fax: $+82-31-219-5238$

E-mail: ee80@hanmail.net

Received: August 19, 2019

Revised: September 22, 2019

Accepted: September 30, 2019
Objective: Skull fractures are one of the most common trauma injuries among children, and pediatric skull fractures are more complex due to incomplete ossification of sutures. Because 3-dimensional computed tomography (3DCT) can provide a more accurate assessment of all skull fracture types that also increase the detection rate of skull fractures, we need to review pediatric skull fractures based on 3DCT. Methods: Two hundred ninety-one patients younger than 12 years were included in this study. We retrospectively studied the correlation of the skull fracture types, location, severity, and accompanying head injuries with surgery rates. Results: The most common skull fracture type was linear (64.6\%), followed by mixed (20.6\%) and diastatic (7.6\%). The most common location of skull fractures was parietal (43.3\%), followed by occipital (26.1\%) and frontal (13.4\%). Thirty-six patients (12.4\%) underwent surgery. Statistically significant difference in surgical risk was observed in the presence of subgaleal hemorrhage, epidural hemorrhage, subdural hemorrhage, intracerebral hemorrhage, subarachnoid hemorrhage, and brain swelling $(p<0.05)$. Conclusion: In most pediatric patients with skull fractures, 3DCT studies seem to be important in terms of diagnostic accuracy for skull fracture types, locations, and risk of surgery. We suggest that skull fracture severity, type, and location with degree of other accompanying head injury lesions may be significant prognostic factors.

Key Words: Craniocerebral trauma; Pediatrics; Skull fractures; Tomography, X-ray computed

\section{INTRODUCTION}

Skull fractures comprise $2 \%$ to $16 \%$ of all pediatric skull fractures and have been reported to be related to intracranial injury such as intracranial hemorrhage and contusion in 15\% to $50 \%$ of patients $3,1,12,13,15)$. Accurate diagnosis of pediatric skull fracture is essential with regard to treatment ${ }^{5}$. However, as the pediatric skull bone is thin and lacks ossification, diagnosis of linear fractures by simple X-ray imaging may be difficult, and sutural ossifications are often incomplete, making accurate detection of diastatic skull fractures (DSF) difficult ${ }^{4,6)}$. To resolve these problems, Orman et al. ${ }^{11)}$ recently proposed the use of 3-dimensional computed tomography (3DCT) for enhanced detection of linear skull fractures in pediatric patients. Furthermore, Sim et al. ${ }^{17)}$ suggested that 3DCT can provide a more accurate diagnosis of not only linear skull fractures but also DSFs. Importantly, owing to the advances in 3DCT technology, there is no increased risk of radiation exposure compared to that with 2-dimensional CT (2DCT); hence, this imaging modality was recommended as the primary diagnostic tool for the assessment of pediatric skull fractures in the emergency rooms'.

As the use of 3DCT has become widespread in the diagnosis of pediatric skull fractures, the diagnostic accuracy of skull fractures has increased, and the detection rates of skull fractures have concomitantly increased as well. DSF, which has been reported rarely to date, is currently the second most frequent after linear skull fractures"). Therefore, it is deemed necessary to reconsider the significance of skull fracture in the present 3DCT era compared to the past reliance on plain X-rays and 2DCT for diagnosis and prognosis of pediatric skull fractures. The objective of this retrospective study was to investigate whether surgical risks are increased with respect to the degree, location, and type of accompanying skull fracture, based on 3DCT data, in 291 pediatric patients with skull fractures. 


\section{MATERIALS AND METHODS}

\section{Patients}

The medical records of 291 pediatric patients younger than 12 years who visited our institute for head trauma and were suspected to have skull fractures and underwent 3DCT, from 2005 to 2014, were retrospectively evaluated. Patients with nontraumatic skull fractures from other etiologies and neonates were excluded from this study. Those who did not undergo 3DCT within $48 \mathrm{hr}$ after head injury were also excluded. A 3DCT was performed as previously described to assess the skull fracture type, location, and severity (Fig. 1). The skull fracture severity was scored for the number of skull fractures and was defined as 1 degree for a total of 1 fracture, 2 degrees for 2 fractures, 3 degrees for 3 fractures, and 4 degrees for $\geq 4$ fractures or more. To compare the clinical characteristics of the pediatric skull fractures, the presence of accompanying
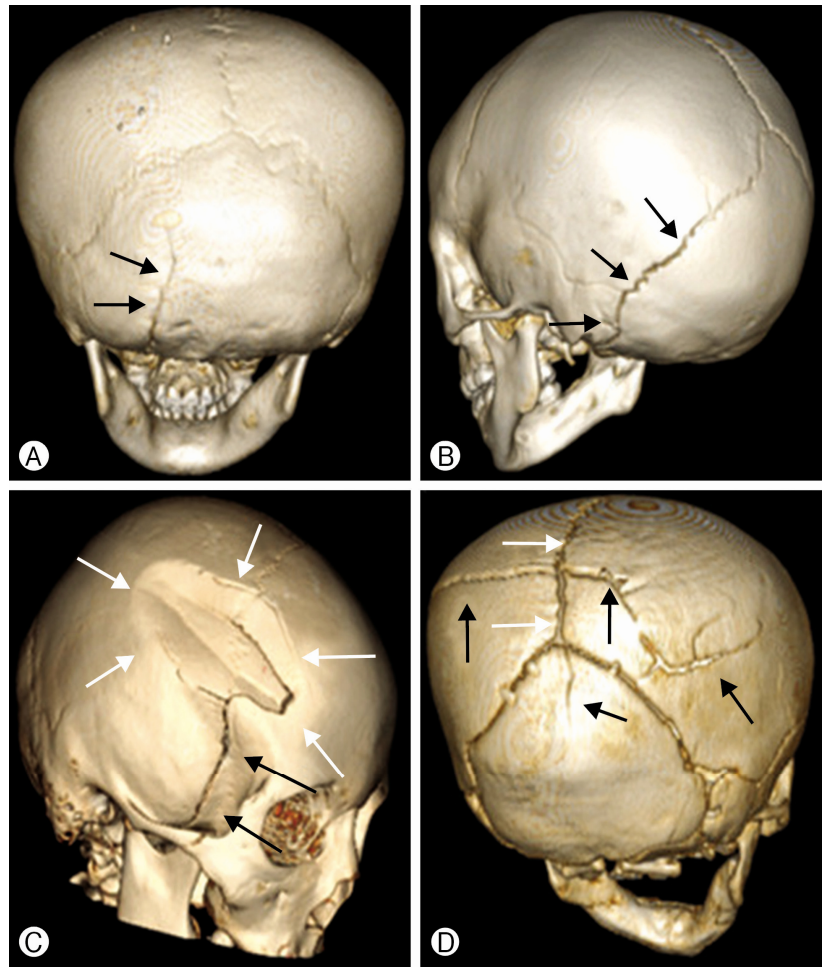

Fig. 1. Skull fracture types on three-dimensional computed tomography. (A) A 47-month-old boy with an occipital linear fracture (black arrows). (B) A 48-month-old boy with a left lambdoid suture diastatic fracture (black arrows). (C) A 129-month-old boy with a compressed fracture involving the right frontal, parietal, and temporal bones (white arrows) and with linear skull fracture (black arrows). (D) A 5-monthold boy with multiple linear fractures involving the parietal and occipital bones (black arrows) and with sagittal diastatic fracture showing widening of the posterior half sagittal suture (white arrows). lesions such as subgaleal hematoma, pneumocephalus, epidural hemorrhage (EDH), subdural hemorrhage (SDH), intracerebral hemorrhage (ICH), subarachnoid hemorrhage (SAH), and brain swelling was also assessed.

\section{Statistical Analyses}

Statistical analyses were performed through $\chi^{2}$ test with 95\% confidence intervals for the comparison of correlation. Univariate and multivariate logistic regression analyses were used to calculate the p-values and odds ratios (ORs) for the accompanying head injuries factors (coding 1 for the head injury group and coding 0 for the no-head-injury group) based on the surgery risk.

\section{RESULTS}

\section{Incidence and Clinical Characteristics of Pediatric Skull Fractures Based on 3DCT}

Among the 291 patients with pediatric skull fractures, 192 were boys and 99 were girls $(\mathrm{p}=0.398)$. The mean age was $5.0 \pm 3.5$ years, with the following age distribution: 0 to 1 years, $142(48.8 \%) ; 2$ to 5 years, 61 (21.0\%); and 6 to 12 years, 88 $(30.2 \%)$. A total of $202(69.4 \%)$ children had single simple fracture, $87(30.0 \%)$ had $\geq 2$ fractures, $60(20.6 \%)$ had 2 multiple fractures, 20 (6.9\%) had 3 fractures, and 9 patients (3.1\%) had $\geq 4$ fractures. Surgery was performed in 36 patients, which comprised $12.4 \%$ of the total 291 patients (Table 1). A total of $3(1.0 \%)$ children with multiple fractures did not undergo surgery and died.

Table 1. Demographic factors of 291 children with skull fractures

\begin{tabular}{lc}
\hline \hline Demographic factors & Value \\
\hline No. of patients & 291 \\
Boys & 192 \\
Girls & 99 \\
Age (year) & $5.0 \pm 3.5$ \\
Age distribution & \\
Infants (0-1 years of age) & $142(48.8)$ \\
Toddlers (2-5 years of age) & $61(21.0)$ \\
Young children (6-12 years of age) & $88(30.2)$ \\
Skull fracture severity & \\
Simple skull fractures & $202(69.4)$ \\
Multiple/Compound skull fractures & $87(30.0)$ \\
Fracture 2 & $60(20.6)$ \\
Fracture 3 & $20(6.9)$ \\
Fracture 4 and more & $9(3.1)$ \\
No. of patients who underwent surgery & $36(12.4)$ \\
\hline
\end{tabular}

The data is presented as number (\%) or mean \pm standard deviation. 
The most common type of skull fracture was linear $(n=188$, 64.6\%), followed by DSF ( $n=22,7.6 \%$ ), depression ( $n=21,7.2 \%$ ), and mixed type $(n=60,20.6 \%)$. Skull fracture locations were in the parietal bone $(126,43.3 \%)$, followed by the occipital bone $(n=76,26.1 \%)$, frontal bone ( $n=39,13.4 \%)$, multiple bones $(n=44,15.1 \%)$, and temporal bone $(n=6,2.1 \%)$.

\section{Accompanying Head Injuries and Surgery}

The most common accompanying lesion among pediatric skull fractures in the 291 children was subgaleal hemorrhage $(\mathrm{n}=243,83.5 \%)$, followed by $\mathrm{EDH}(\mathrm{n}=100,34.4 \%)$, pneumocephalus ( $n=48,16.5 \%)$, SDH ( $n=11,3.8 \%)$, ICH ( $n=8,2.7 \%)$, SAH $(n=5,1.7 \%)$, and brain swelling $(n=5,1.7 \%)$. Of the 291 children, 36 (12.4\%) underwent 39 surgeries, which consisted of craniotomy and removal of intracranial hemorrhage in 22, followed by craniotomy and removal of foreign bodies or reduction of skull bones in 5, and 3 decompressive craniectomy and burr hole operation each. Three children underwent operations twice: one underwent removal of intracranial hemorr-

Table 2. Correlation of skull fracture characteristics with surgical risk in 291 children with skull fractures

\begin{tabular}{lrc}
\hline \hline Factors & Operation ratio & p-value \\
\hline Skull fracture severity & & $<0.001$ \\
1 & $14 / 202(6.9 \%)$ & \\
2 & $11 / 60(18.3 \%)$ & \\
3 & $8 / 20(40.0 \%)$ & \\
4 & $3 / 9(33.3 \%)$ & \\
Skull fracture types & & $<0.001$ \\
Linear & $12 / 188(6.4 \%)$ & \\
Diastatic & $3 / 22(13.6 \%)$ & \\
Depressed & $4 / 21(19.0 \%)$ & \\
Multiple & $17 / 60(28.3 \%)$ & \\
Skull fracture locations & & 0.030 \\
Frontal & $7 / 39(17.9 \%)$ & \\
Parietal & $10 / 126(7.9 \%)$ & \\
Occipital & $7 / 76(9.2 \%)$ & \\
Temporal & $1 / 6(16.7 \%)$ & \\
Multiple & $11 / 44(25.0 \%)$ & \\
\hline p-value by $x^{2}$ test. &
\end{tabular}

hage surgery followed by decompressive craniectomy, one underwent removal of intracranial hemorrhage surgery followed by burr hole operation, and one underwent decompressive craniectomy surgery followed by burr hole surgery.

\section{Correlation of Skull Fracture Characteristics and Accompanying Head Injuries with Operation Risk}

We analyzed and compared the surgical risk and accompanying head injuries such as subgaleal hematoma, pneumocephalus, $\mathrm{EDH}, \mathrm{SDH}, \mathrm{ICH}, \mathrm{SAH}$, and brain swelling of the skull fracture characteristics in terms of skull fracture type, location, and severity. Our results showed that all 3 skull fracture characteristics (skull fracture type, location, and severity) demonstrated statistical significance and that subgaleal hematoma, $\mathrm{EDH}, \mathrm{SDH}, \mathrm{ICH}, \mathrm{SAH}$, and brain swelling (with the exception of pneumocephalus) showed statistical significance on the $\chi^{2}$ test (Table 2).

Risk comparison by univariate logistic regression revealed that surgery risk in accompanying head injuries increased 7.5-fold with EDH, 6.7-fold with SDH, and 10.3-fold with brain swelling (Table 3). Risk comparison by multivariate logistic regression revealed that surgery risk in accompanying head injuries only increased 7.5-fold with EDH (Table 3).

\section{DISCUSSION}

According to previous studies, boys are more prone to pediatric skull trauma than girls, and our results show a similar ratio of $1.94: 1$, in favor of male children ${ }^{7,8,10)}$.

The incidence rate of skull fractures among pediatric head trauma ranged from $42 \%$ to $72 \%$, whereas previous studies have shown a $64 \%$ to $73 \%$ incidence rate of linear skull fractures, which is similar to our results ${ }^{1,7-9,16,18)}$. However, past investigations regarding DSF did not perform diagnostic 3DCT such that the diagnostic accuracy was markedly lower (1\%) than our rate of $7.6 \%{ }^{18)}$. Regarding multiple fractures, the previously reported incidence of $26 \%$ is similar to our observed incidence of $21 \%{ }^{18)}$.

With respect to skull fracture location, Harwood-Nash et $\mathrm{al}^{7)}$ reported that among 1,187 pediatric patients with skull fractures, the parietal bone was most the commonly frac-

Table 3. Significant results in the univariate and multivariate logistic regression models of accompanying head injuries and the surgery risks in 291 children with skull fractures

\begin{tabular}{llccr}
\hline \hline Logistic regression & Head injury factors & $\mathrm{B} \pm \mathrm{SE}$ & $\mathrm{p}$-value & $\mathrm{OR}(95 \% \mathrm{Cl})$ \\
\hline \multirow{3}{*}{ Univariate } & Epidural hemorrhage & $2.012 \pm 0.409$ & 0.000 & $7.479(3.355-16.675)$ \\
& Subdural hemorrhage & $1.901 \pm 0.635$ & 0.003 & $6.694(1.929-23.226)$ \\
Multivariate & Brain swelling & $2.332 \pm 0.887$ & 0.009 & $10.298(0.810-58.581)$ \\
\hline
\end{tabular}

B: estimate; SE: standard error; OR: odds ratio; Cl: confidence interval. 
tured bone (40\%), followed by the occipital (30\%), frontal $(22 \%)$, and temporal bones (8\%). In the present investigation, we also showed that the parietal area was the most commonly affected skull fracture location (43.3\%), followed by occipital (26.1\%), frontal (13.4\%), and temporal bones (2.1\%).

The incidence of subgaleal hematoma and pneumocephalus in pediatric head trauma was shown to be approximately $74 \%$ and $14 \%$ to $15 \%$, respectively, which is similar to our current study incidence of $83.5 \%$ and $16.5 \%$, respectively ${ }^{17,18)}$.

Similarly, the previously reported incidence of EDH was $26.5 \%$ to $38.6 \%$, in agreement with our observed incidence of $34.4 \%{ }^{1,14,18)}$. Bhutta et al. ${ }^{1)}$ showed that in 34 children with head trauma due to all-terrain vehicle injuries, $8(23.5 \%)$ had accompanying SDH and 13 (38.2\%) had brain hemorrhage, which are markedly higher than those observed in the present study, with $3.8 \%$ of $\mathrm{SDH}$ and $2.7 \%$ of brain hemorrhage. In this respect, we propose that as the number of children in the study of Bhutta et al. ${ }^{1)}$ was small, further statistical comparison is warranted.

The incidence of surgery for pediatric head injury ranged from $1.4 \%$ to $17.5 \%$ in the literature, which is similar to that observed in our study (12.4\%), whereas the mortality rate in pediatric head injury has been reported as $6 \%$ to $8 \%$, which is in contrast to our far lower rate of $1 \% 0^{2,8,9)}$. The possible reason for our comparatively lower mortality rate may be because we did not focus our research on the mortality rate of all pediatric patients with skull trauma, but instead limited the study to those children with skull fractures who underwent 3DCT; as such, we did not perform time-consuming 3DCT for patients with very severe head injury.

With regard to pediatric external trauma that leads to skull fractures, Bonfield et al. ${ }^{2)}$ demonstrated that $7.5 \%$ underwent surgery and observed that skull fractures that consisted of 2 skull bones were $22 \%$ and those with 3 skull bones were $31.3 \%$. They concluded that more severe skull fracture injuries have higher surgery risk. In a study by Shah et al. ${ }^{16)}$, calvarial fractures were observed to be significantly related to brain injuries, epidural hematoma, and $\mathrm{SDH}$. In the same study, brain injuries were observed to be significantly related to longterm disability or death. Schutzman and Greenes ${ }^{15)}$ revealed that $15 \%$ to $30 \%$ of linear skull fractures were related to intracranial injury, and Quayle et al. ${ }^{12)}$ reported that the likelihood of an intracranial injury is increased 4-fold in the presence of skull fractures. In contrast, Bhutta et al. ${ }^{1)}$ revealed no association of brain injury and skull fracture in 27 children with all-terrain vehicle injuries that led to brain injury, which was also supported by Godano et al. $^{4}$, who reported no relationship between age and Glasgow Coma Scores in 62 pediatric patients with skull trauma. In the present study, we observed statistically significant results between surgical risk and skull fracture type, location, and severity and between surgical risk and $\mathrm{EDH}$, among accompanying head injuries, on $\chi^{2}$ test and univariate and multivariate logistic regression analyses.

\section{CONCLUSION}

The diagnostic accuracy of skull fracture type and location is enhanced by the utilization of 3DCT, which can assist in treatment decisions for pediatric patients with head injury. Statistically significant results were obtained between surgical risk and skull fracture type, location, severity, and presence of accompanying EDH.

\section{ACKNOWLEDGMENTS}

We are grateful to Dr. K.H. Chang for preparing the English version of the manuscript.

\section{CONFLICTS OF INTEREST}

No potential conflict of interest relevant to this article was reported.

\section{REFERENCES}

1. Bhutta ST, Greenberg SB, Fitch SJ, Parnell D: All-terrain vehicle injuries in children: injury patterns and prognostic implications. Pediatr Radiol 34:130-133, 2004

2. Bonfield CM, Naran S, Adetayo OA, Pollack IF, Losee JE: Pediatric skull fractures: the need for surgical intervention, characteristics, complications, and outcomes. J Neurosurg Pediatr 14: 205-211, 2014

3. Dunning J, Daly JP, Lomas JP, Lecky F, Batchelor J, MackwayJones K: Derivation of the children's head injury algorithm for the prediction of important clinical events decision rule for head injury in children. Arch Dis Child 91:885-891, 2006

4. Godano U, Serracchioli A, Servadei F, Donati R, Piazza G: Intracranial lesions of surgical interest in minor head injuries in paediatric patients. Childs Nerv Syst 8:136-138, 1992

5. Greenes DS, Schutzman SA: Clinical significance of scalp abnormalities in asymptomatic head-injured infants. Pediatr Emerg Care 17:88-92, 2001

6. Grossart KW, Samuel E: Traumatic diastasis of cranial sutures. Clin Radiol 12:164-170, 1961

7. Harwood-Nash DC, Hendrick EB, Hudson AR: The significance of skull fractures in children. A study of 1,187 patients. Radiology 101:151-156, 1971

8. Kim KA, Wang MY, Griffith PM, Summers S, Levy ML: Analysis of pediatric head injury from falls. Neurosurg Focus 8:e3, 2000

9. Levi L, Guilburd JN, Linn S, Feinsod M: The association between skull fracture, intracranial pathology and outcome in pediatric head injury. Br J Neurosurg 5:617-625, 1991

10. Mangano FT, Menendez JA, Smyth MD, Leonard JR, Narayan P, Park TS: Pediatric neurosurgical injuries associated with allterrain vehicle accidents: a 10-year experience at St. Louis 
Children's Hospital. J Neurosurg 105:2-5, 2006

11. Orman G, Wagner MW, Seeburg D, Zamora CA, Oshmyansky A, Tekes A, et al.: Pediatric skull fracture diagnosis: should 3D CT reconstructions be added as routine imaging? J Neurosurg Pediatr 16:426-431, 2015

12. Quayle KS, Jaffe DM, Kuppermann N, Kaufman BA, Lee BC, Park TS, et al.: Diagnostic testing for acute head injury in children: when are head computed tomography and skull radiographs indicated? Pediatrics 99:E11, 1997

13. Ringl H, Schernthaner RE, Schueller G, Balassy C, Kienzl D, Botosaneanu A, et al: The skull unfolded: a cranial CT visualization algorithm for fast and easy detection of skull fractures. Radiology 255:553-562, 2010

14. Sarkar K, Keachie K, Nguyen U, Muizelaar JP, ZwienenbergLee $\mathrm{M}$, Shahlaie K: Computed tomography characteristics in pediatric versus adult traumatic brain injury. J Neurosurg Pediatr 13:307-314, 2014

15. Schutzman SA, Greenes DS: Pediatric minor head trauma. Ann Emerg Med 37:65-74, 2001

16. Shah CC, Ramakrishnaiah RH, Bhutta ST, Parnell-Beasley DN, Greenberg BS: Imaging findings in 512 children following allterrain vehicle injuries. Pediatr Radiol 39:677-684, 2009

17. Sim SY, Kim HG, Yoon SH, Choi JW, Cho SM, Choi MS: Reappraisal of pediatric diastatic skull fractures in the 3-dimensional CT era: Clinical characteristics and comparison of diagnostic accuracy of simple skull X-ray, 2-dimensional CT, and 3-dimensional CT. World Neurosurg 108:399-406, 2017

18. Zulfiqar M, Kim S, Lai JP, Zhou Y: The role of computed tomography in following up pediatric skull fractures. Am J Surg 214: 483-488, 2017 